\title{
Konvergenz von Lohnentwicklung und Makropolitik für eine stabile Europäische Wirtschafts- und Währungsunion
}

Die Krise des Euroraums hat massive Lücken im wirtschaftspolitischen Regelungsrahmen (economic governance) der Wirtschafts- und Währungsunion (WWU) aufgedeckt. Die Vielzahl von Reformvorschlägen aus Wissenschaft und Politik zur Schließung dieser Lücken richtet sich primär auf Finanzwirtschaft und Fiskalunion. Dabei ist die Krise in erster Linie das Ergebnis kumulativer Divergenzen zwischen den Mitgliedstaaten, und zwar sowohl hinsichtlich der Lohn- und Preisentwicklung als auch der Binnennachfrage und Leistungsbilanz. Der Beitrag entwickelt zum einen Maßnahmen, um eine nachhaltige, gleichgewichtige realwirtschaftliche Entwicklung der Mitgliedstaaten und der WWU zu ermöglichen. Zum anderen werden eine wissenschaftliche Politikberatung und eine daran orientierte kooperative Ausrichtung von Geld-, Fiskal- und Lohnpolitik im Rahmen geeigneter Institutionen empfohlen.

WILLI KOLL, ANDREW WATT

\section{Das Fundament der WWU - Trotz vieler Reformen noch schwerwiegende Mängel}

Die vergangenen Jahre waren mit Blick auf die Europäische Union (EU) von heftigen Auseinandersetzungen um deren Zukunft gekennzeichnet. Der Streit ging darum, ob die EU - und insbesondere die Wirtschafts- und Währungsunion (WWU) - ihren inneren Zusammenhalt lockern, teilweise gar auflösen oder im Gegenteil vertiefen sollte. Dieser Beitrag beschäftigt sich mit den Voraussetzungen für eine erfolgreiche Wirtschafts- und Fiskalunion als Fundament der Währungsunion. Denn ohne stabile wirtschaftliche Entwicklung hat zum Beispiel eine Bankenunion keinen Erfolg. Auch werden sozialpolitische Zielsetzungen kaum einlösbar sein. Nachhaltig wird die WWU indes nur sein, wenn sie in ihren Politiken dem Umweltschutz, sei es präventiv oder korrigierend, die Priorität einräumt, die er schon angesichts der bisherigen dramatischen Entwicklungen verdient (Koll 2016).

Blickt man auf die Reformbemühungen nach der Krise (so z. B. Watt/Watzke 2018; Dullien 2018; Herr et al. 2019), sieht man zunächst eine beeindruckende Anzahl an Initiativen, Instrumenten und neuen Institutionen. Die Krise des Euroraums ist aber bei Weitem nicht überstanden; kritische Schwächen bleiben. Ohne alle Refor- men besprechen zu können, sollen an dieser Stelle vier kritische Unzulänglichkeiten herausgearbeitet werden, die sich wie folgt zusammenfassen lassen: Der Fokus der Reformen lag überwiegend auf der Finanzwirtschaft und dem Bankensektor und - weiterhin - auf den Salden der öffentlichen Haushalte. Reformempfehlungen litten an einem ungerechtfertigten Glauben an die ausschließliche und auch makroökonomisch ausreichende Effektivität von angebotsseitigen Strukturreformen und - so paradox es klingen mag - an einer Fokussierung auf den Euroraum zulasten der nationalen Ebene.

(1) Viele einflussreiche Analysen identifizierten die doom loop (Todesschleife) zwischen dem nationalen Bankensektor und dem jeweiligen öffentlichen Haushalt als Achilles-Ferse des Euroraums (ein prominentes Beispiel ist Bénassy-Quéré et al. 2018). Bankenrettungen überfordern Nationalstaaten, die nicht mehr auf eine nationale Zentralbank als Kreditgeber der letzten Instanz (lender of last resort) zurückgreifen können. Zweifel an der Nachhaltigkeit der Staatsfinanzen entwerten andererseits die Staatspapiere als wichtige Posten auf der Aktivseite der Bankbilanzen, was wiederum die Banken anfällig macht.

Als Antwort sind weitreichende Pläne für eine Bankenunion veröffentlicht und in nicht unerheblichen Maße auch umgesetzt worden. (Der wichtige Schritt hin zu einer soliden gesamteuropäischen Einlagensicherung steht allerdings noch aus.) Dies ist alles zu begrüßen. Wie wir 
aber zeigen werden, ist die doom loop zwar ein wichtiger Krisenbeschleuniger. Sie ist aber keine eigentliche Ursache der Krise. Die finanzwirtschaftliche Instabilität fußt auf grundsätzlichen realwirtschaftlichen Instabilitäten und Ungleichgewichten.

(2) Ähnliches lässt sich für die Fokussierung auf die Defizite und Schulden der öffentlichen Haushalte feststellen. In einer Währungsunion mit zentraler Geldpolitik kann es keine ausschließlich nationale finanzpolitische Autonomie geben, erst recht nicht, wenn es Maßnahmen der Risikoteilung bzw. Gesamthaftung gibt und geben soll. Es muss Regeln geben, und sie müssen auch durchgesetzt werden. Jedoch waren die vorherrschende Fixierung der Krisenanalyse auf mangelnde fiskalische Verantwortung und die daraus abgeleitete einseitige und im Laufe der Krise verschärfte Überwachung von nominalen wie strukturellen Defiziten und von Schuldenständen falsch und hatten fatale Konsequenzen. ${ }^{1}$

Wie wir zeigen werden, muss vielmehr die nationale Fiskalpolitik darauf angelegt werden, makroökonomischen Ungleichgewichten und über- bzw. unterschießenden Inflationsraten rechtzeitig und „symmetrisch“ entgegenzuwirken.

(3) Seit Beginn der Eurozone war die Agenda von Rat und Kommission neben der Fokussierung auf Fiskalsalden auf Strukturreformen als primäre Politik für Wachstum und Beschäftigung gerichtet. Sie waren zentrales Element der länderspezifischen Empfehlungen an die Mitgliedstaaten, aber auch länderübergreifender Konzepte wie der „Strategie Europa 2020“ und des „Euro-Plus-Pakts“.

Strukturreformen - wenn sie zielführend ausgewählt und ausgestaltet sind - erhöhen und verbessern das Produktionspotenzial der Volkswirtschaft, indem sie quantitativ die Grenzen möglicher Expansion hinausschieben und qualitativ Innovationen befördern.

Entscheidend ist jedoch, dass diese Potenziale auch aktiviert werden. So werden z.B. Innovationen wesentlich durch Investitionen umgesetzt. Investitionen brauchen aber neben einer Rentabilitätserwartung auch eine Absatzerwartung, um realisiert zu werden. Diese Absatzerwartungen müssen aber durch nachfrageseitig wirkende makroökonomische Instrumente wie Geld-, Währungs- und Fiskalpolitik, aber auch eine gleichgewichtige Einkommensverteilung realisiert werden. Ohne diese nachfrageseitige Flankierung führen sie bloß zu einer Kostenreduzierung und damit außenwirtschaftlich zu einer beggar-thy-neighbour-Politik: Der zusätzlichen Nachfrage für inländische Produzenten stehen entsprechenden Verluste bei Marktanteilen, Einkommen und Beschäftigung in anderen Mitgliedstaaten entgegen.

Mit anderen Worten: Geeignete Strukturreformen können den Einsatz makroökonomischer Instrumente in ihrem Wirkungsgrad erhöhen, sie aber nicht ersetzen. Insofern bedarf die bisher einseitige Orientierung der Politikausrichtung nicht nur auf Ebene der Eurozone, sondern auch in den Mitgliedstaaten der Ergänzung durch einen stabilitäts-, wachstums- und beschäftigungsorientierten makroökonomischen Policy-Mix. Daher überrascht es nicht, dass etwa die LABREF-Database der EU-Kommission tausende von Arbeitsmarktreformen in den Mitgliedstaaten erfasst oder die OECD ihren europäischen Mitgliedstaaten eine wesentliche Reduzierung der Regulierung der Gütermärkte attestiert, die grundsätzliche Problematik der Währungsunion aber ungelöst bleibt.

(4) Seit Beginn der Währungsunion sind primär auf Ebene der Eurozone eine Vielzahl von Institutionen, Regelungen und Prozessen etabliert worden. ${ }^{2}$ Sie haben im Ergebnis nicht ausgereicht, die gravierende Krise der Eurozone zu vermeiden. Nach der Krise hat sich diese Tendenz eher noch verstärkt. Bis zuletzt hat es - beginnend mit dem Bericht der fünf Präsidenten zur Vollendung der Wirtschaftsund Währungsunion im Jahr 2015 über das Weißbuch der Kommission zur Zukunft Europas im Mai 2017 bis hin zur Vorlage eines „Fahrplans“ für die Vertiefung der WWU Ende 2017 - vielfältige Vorschläge für eine Wirtschafts-, Finanz-, Fiskal-, Sozial- und politische Union gegeben, die fast alle auf die europäische Ebene gerichtet sind.

Ein Grundprinzip des EU-Vertrags ist Subsidiarität. Das bedeutet zum einen, insbesondere im Falle einer Notlage eines Mitgliedstaates, dass ihm die anderen Mitgliedstaaten und die Institutionen und Instrumente auf EU-Ebene zu Hilfe kommen. Das bedeutet aber auch, dass zuerst auf Ebene der Mitgliedstaaten nach Ursachen und Lösungen gesucht werden muss. Genau daran hat es gefehlt, wie im Weiteren gezeigt wird.
1 “Drawing lessons from the crisis, we need to broaden the focus of our surveillance beyond fiscal policy to include relevant developments of macroeconomic imbalances ..." (European Commission 2010, S. 6; siehe ebd., S. 38: Beschluss der Eurogruppe). - „Es war ein Fehler, dass wir vor allem auf die Staatsfinanzen geschaut haben. Wir hätten ein breiteres Spektrum makroökonomischer Parameter überwachen sollen. [...] Die Entwicklung der Lohnstückkosten, Leistungsbilanzdefizite, Immobilienblasen. Die
Kommission hätte ein Mandat gebraucht, gegen diese Ungleichgewichte genauso vorzugehen wie gegen zu hohe Staatsverschuldung." (Regling 2010, S. 2)

2 Eine Ausnahme ist hier das Verfahren zur Vermeidung und Korrektur makroökonomischer Ungleichgewichte, das sich - wie schon der Stabilitäts- und Wachstumspakt - ausschließlich an die Mitgliedstaaten wendet. 


\section{Schlüsselrolle für Lohn- und Preisentwicklung auf nationaler Ebene}

Für die Lösung der Krise und die Vermeidung einer Wiederholung nimmt die nationale Ebene eine Schlüsselrolle ein. Auf den ersten Blick erscheint das paradox: Wie soll mehr Integration durch einen Rückgriff auf die Ebene der Mitgliedstaaten entstehen?

Die Antwort liegt in einem wichtigen, aber unterbelichteten Merkmal der Krise. Es war keine Krise des Euroraums als Ganzem. Vor der Krise entwickelten sich die wichtigsten wirtschaftlichen Größen wie Wachstum, Beschäftigung, Preisstabilität und außenwirtschaftliches Gleichgewicht im Aggregat der Eurozone eher positiv. Aber diese günstige Entwicklung war nur der Durchschnittswert von nationalen Entwicklungen, die schon recht früh permanent und persistent auseinanderliefen bis hin zu ihrer krisenhaften Zuspitzung.

Am stärksten fielen hier die Unterschiede in der Leistungsbilanz ins Auge: Massiven Defiziten einzelner Mitgliedstaaten standen ebenso massive Überschüsse anderer gegenüber. Ebenso prägnant waren die entsprechenden Divergenzen in der Kapitalbilanz. Die Defizitländer realisierten eine massive Verschuldung, während die Überschussländer entsprechend hohe Auslandsguthaben anhäuften. Als die Finanzmarktkrise ausbrach, kamen diese Kapitalflüsse ruckartig zum Erliegen. Inzwischen haben sich die Divergenzen teilweise zurückgebildet; nach wie vor besteht aber noch weiterer Anpassungsbedarf. ${ }^{3}$

Für eine wirksame Krisenprävention ist es unabdingbar zu erkennen, wie es zu derart gravierenden und krisenauslösenden Divergenzen kommen konnte. Deshalb wird im Folgenden versucht, die Wirkungskanäle, die die Leistungsbilanzentwicklung und ihre Divergenzen bestimmen, nachvollziehbar abzuleiten.

Leistungsbilanz- und Kapitalbilanzsalden sind das finale Ergebnis einer längeren Kette gesamtwirtschaftlicher Prozesse. Die Leistungsbilanz bildet den Strom der Waren und Dienstleistungen in Form von Exporten und Importen ab. Exporte und Importe wiederum sind das Ergebnis mengenmäßiger und preislicher Entwicklungen zwischen den Handelspartnern.

Dabei hängen die Exporte einerseits wesentlich von der Nachfrage und damit der Wachstumsdynamik der Weltwirtschaft ab; diese Einflussgrößen sind zumindest kurzfristig kaum durch nationale Politik zu beeinflussen. Andererseits spielt die preisliche Wettbewerbsfähigkeit (bei vergleichbarer Produktqualität) als Zusammenspiel nationaler und weltweiter Preisentwicklung ebenfalls eine wichtige Rolle.

Die Importe werden in ihrer preislichen Komponente gleichermaßen durch die Relation von Weltmarkt- und nationalen Preisen mitbeeinflusst. Mengenmäßig wird ein Teil, der sogenannte Importgehalt der Exporte, ebenfalls durch den Weltmarkt beeinflusst. Der überwiegende Teil der Mengenkomponente wird jedoch durch die Binnennachfrage, d.h. durch die nationale Entwicklung bestimmt.

Aus diesem Befund ergibt sich die nächste Frage: Was bestimmt den nationalen Anteil an der preislichen Wettbewerbsfähigkeit und was die Binnennachfrage?

Die preisliche Wettbewerbsfähigkeit wird neben den Preisen aller Handelspartner von den Preisen des jeweiligen exportierenden Landes beeinflusst, modifiziert durch Veränderungen des nominalen Wechselkurses. Innerhalb der Währungsunion gibt es jedoch keine Wechselkurse. Die Exportpreise setzen sich demnach zusammen aus den Lohnstückkosten, dem Stückgewinn und dem Abgabesatz (Steuern und Sozialabgaben). Empirisch haben hier in mittelfristiger Betrachtung die Lohnstückkosten und ihre Veränderung - als Differenz von Nominallöhnen und (Arbeits-)Produktivität - das größte Gewicht. Dabei wird die Produktivität als eine Komponente durch die Qualität der Arbeit, die Kapitalintensität der Produktion und durch die konjunkturelle Auslastung der Kapazitäten bestimmt. Dieses sind alles Größen, die entweder nur mittelfristig zu beeinflussen oder - wie die Konjunktur ihrerseits - das Ergebnis des Zusammenwirkens makroökonomischer Einflussgrößen sind.

Dagegen bestimmt sich die Lohnkomponente unmittelbar als Ergebnis der Nominallohnentwicklung, d.h. von Tarifvereinbarungen (unter Berücksichtigung der sogenannten Lohndrift).

Die Binnennachfrage als Konsum- und Investitionsnachfrage wird einerseits exogen von der Ausrichtung der Geld- und Währungs- sowie der Fiskalpolitik beeinflusst. In einer Währungsunion ist dabei die monetäre Politik für alle Mitgliedstaaten gleich, in dem Sinne, dass es einen einheitlichen kurzfristigen Nominalzins und Außen-Wechselkurs gibt. Andererseits wirkt die Verwendung des gesamtwirtschaftlichen Einkommens in Form von Konsum oder Sparen auf die Binnennachfrage. Die Konsum- und Sparquote wird dabei wesentlich von der Verteilung des Einkommens vorgegeben. Diese unterliegt in der Regel kurzfristig geringeren Schwankungen nach oben und unten und ändert sich eher in mittelfristiger Sicht.

Damit bleibt die Lohn- und Preisentwicklung eine wesentliche kurzfristige Determinante auch der Binnennachfrage. Denn sie bestimmt entscheidend über die Höhe des Realzinses. Der Realzins als Differenz zwischen Nominalzins und Preissteigerungsrate ist aber eine zentrale wirtschaftliche Größe. Niedrige Realzinsen stärken die gesamtwirtschaftliche Binnennachfrage über mehrere Kanäle. Sie reduzieren zum einen den Anreiz zu sparen,

3 Eine detaillierte Analyse länderspezifischer Entwicklungen findet sich bei Bibow/Flassbeck (2018). 
und erhöhen die Neigung zu konsumieren. Zum anderen machen sie Investitionen rentabel, die bei höherem Realzins nicht getätigt werden würden. Das gilt insbesondere für den stark zinsreagiblen Bausektor. Dabei wird angenommen, dass der langfristige Realzins durch den kurzfristigen Realzins wesentlich beeinflusst wird. In den Mitgliedstaaten der WWU ergibt sich der kurzfristige Realzins als Differenz von gemeinsamem Nominalzins der Europäischen Zentralbank (EZB) und der länderspezifischen Preisentwicklung. Diese wird ihrerseits von den Lohnstückkosten und damit von den Nominallöhnen beeinflusst und wirkt auf diese zurück. Dadurch entstehen sog. Nominallohn-Preis-Spiralen, die sich unterschiedlich schnell drehen.

Wichtig für ihre Wirkung auf Binnennachfrage und Leistungsbilanz ist, dass z. B. ein Anstieg der Lohnstückkosten im Vergleich zu einem anderen Mitgliedstaat über einen entsprechenden relativen Anstieg der gesamtwirtschaftlichen Preisentwicklung zugleich den Realzins senkt als auch die preisliche Wettbewerbsfähigkeit verschlechtert. Letztere lässt die Importe steigen und dämpft gleichzeitig die Exporte. Relativ gesunkene Realzinsen wiederum wirken expansiv auf die Binnennachfrage und damit auch expansiv auf den Import. Per Saldo kommt es zu einer kumulativen Passivierung der Leistungsbilanz. Im Falle relativ gesunkener Lohnstückkosten tritt das Gegenteil ein: eine starke Aktivierung der Leistungsbilanz.

Verschärft wird diese Problematik von einseitig wirkenden fiskalischen Regeln. Länder mit niedrigen Realzinsen und höherer Inflation haben dank höheren Wachstums des nominalen Bruttoinlandsprodukts (BIP) kaum Probleme, die fiskalischen Regeln des Stabilitäts- und Wachstumspakts (SWP) einzuhalten. Sie neigen deshalb zu einer expansiven Fiskalpolitik, die damit prozyklisch Binnen- und Importnachfrage verstärkt. Restriktiv auf Binnendynamik und Importe und damit ebenfalls, wenn auch in andere Richtung, prozyklisch und problemverschärfend, wirken die Regeln bei Ländern mit hohen Realzinsen und niedriger Inflation.

Im Ergebnis beeinflusst die Lohnentwicklung über die Lohnstückkosten sowohl die preisliche Wettbewerbsfähigkeit als auch die Binnennachfrage. Sie ist damit - im Zusammenwirken mit einer prozyklischen Fiskalpolitik - ein entscheidender Faktor in der Entstehung krisenhafter Divergenzen.

Was bestimmt nun die Lohnentwicklung? Neben der jeweiligen konjunkturellen Situation insbesondere am Arbeitsmarkt ist sie abhängig von ihrer institutionellen Form. Hierzu gibt es eine ausufernde Literatur (vgl. die Diskussion in Watt 2017, Kapitel 3). Für unseren Zusammenhang ist wesentlich, dass zentralisierte bzw. koordinierte Systeme der Lohnfindung die Möglichkeiten der Sozialpartner erhöhen, (Nominal-)Lohnänderungen unabhängig von der kurzfristigen konjunkturellen Entwicklung festzulegen und mittelfristig mehr im Einklang mit der im nächsten Abschnitt beschriebenen Goldenen Regel auszurichten. In stark dezentralen Systemen hängt dagegen die Verhandlungsposition der Arbeitnehmer stark von der jeweiligen Entwicklung des Unternehmensertrags ab. Dieser wiederum wird wesentlich durch die gesamtwirtschaftliche Entwicklung beeinflusst. Da, wie oben gezeigt, die Löhne ihrerseits einen starken Einfluss auf die Konjunktur haben, neigt dezentrale Lohnfindung dazu, stärker „prozyklische“ Wirkungen zu entfalten, d.h. konjunkturelle Ausschläge zu verstärken.

\section{Empirische Evidenz: Divergenzen und Krise im Euroraum}

Die theoretisch abgeleiteten Zusammenhänge zeigen sich auch in der Empirie: Die Mitgliedstaaten sind 1999 mit einem großen Unterschied in der konjunkturellen Dynamik in die WWU eingetreten. Einige Länder haben durch die beim Eintritt stark gesunkenen Realzinsen einen ebenso starken konjunkturellen Impuls erhalten. Andere Länder sind mit einer relativ schwachen Dynamik in die WWU gestartet. Wie aus den vorangegangenen Überlegungen zu erwarten war, hat sich diese Divergenz auch in der Lohn- und damit auch Preisentwicklung in den folgenden Jahren fortgesetzt und ihrerseits wiederum die Schere in der konjunkturellen Entwicklung zwischen den Mitgliedstaaten weiter geöffnet. Verstärkt wurden diese Divergenzen zudem durch eine tendenziell prozyklische Fiskalpolitik: Dort, wo durch den Boom bedingt hohe Mehreinnahmen entstanden, wurden diese auch wieder ausgegeben. Binnen- und damit auch Importnachfrage und Defizite in der Leistungsbilanz weiteten sich zusätzlich aus. In Ländern mit schwacher konjunktureller Dynamik wie z. B. Deutschland wurden unter dem Druck der fiskalischen Regeln Steuern erhöht und/oder Ausgaben gekürzt, was hier zu einer weiteren Dämpfung der gesamtwirtschaftlichen Entwicklung führte.

Das Ergebnis war eine permanente und persistente jährliche Auseinanderentwicklung von Binnendynamik sowie Leistungs- und Kapitalbilanz (Abbildung 1, unten). Diese Divergenzen haben sich über fast ein Jahrzehnt in einer Höhe kumuliert, die aufgrund der drastisch erhöhten Sensibilität nach Ausbruch der Finanzmarktkrise plötzlich nicht mehr als nachhaltig und tragbar erschienen. Die Reaktion war ein schockartiger Einbruch in der realwirtschaftlichen und finanzwirtschaftlichen Entwicklung in einigen Mitgliedstaaten, der sich in einer tiefen Krise manifestierte.

Die Lohnentwicklung stand dann auch mit im Zentrum beim Versuch, die Divergenzen insbesondere in der Leistungsbilanz abzubauen, allerdings mit sehr einseitiger Stoßrichtung. Gepaart mit fiskalischer Austerität zwang die Troika aus Internationalem Währungsfonds (IWF), 
Europäischer Zentralbank (EZB) und EU-Kommission die so genannten Programmländer zu einer starken Senkung der Lohnkosten und Sozialausgaben, auch durch massive Entlassungen. Das Ergebnis war, wie zu erwarten, ein drastischer Einbruch der Binnennachfrage sowie ein schockartiger Anstieg der Arbeitslosigkeit. Die Leistungsbilanz reagierte auf diesen Rückgang der Dynamik mit einer entsprechenden Rückbildung des Defizitsaldos, zunächst jedoch primär über einen Rückgang der Importe als Folge des Einbruchs der Binnendynamik (Abbildung 1, unten).

Erst viel später forderten die gleichen Institutionen auch einen Anpassungsbeitrag in den Überschussländern über eine expansivere Ausrichtung der Fiskalpolitik oder höhere Löhne ein. Auch hier zeigte sich tendenziell, wenn auch viel zu schwach ausgebildet, der zu erwartende Effekt: Binnennachfrage und Importe gewannen an Stärke, die permanente und starke Ausweitung des Leistungsbilanzüberschusses verlangsamte sich und kam in Deutschland zuletzt zum Stillstand, wenngleich nach wie vor weit über dem vorgeschriebenen Schwellenwert der Verordnung zur Vermeidung und Korrektur makroökonomischer Ungleichgewichte.

\section{Lohnfindung und Preisbildung für Stabilität und Konvergenz}

Um eine erneute Krise zu vermeiden, müssen sich Löhne und Preise so entwickeln, dass gravierende Divergenzen bei Realzinsen und preislicher Wettbewerbsfähigkeit zwischen den Mitgliedstaaten vermieden werden. ${ }^{4}$

Das setzt erstens voraus, dass das gesamtwirtschaftliche Preisniveau mittelfristig im Gleichschritt mit den Lohnstückkosten wächst; zweitens müssen alle Mitgliedstaaten in der Lohn- und Preisentwicklung das Preisstabilitätsziel der Geldpolitik beachten, d. h. dass beide Größen mit der Rate des Preisziels der Geldpolitik wachsen. Auf eine einfache Formel gebracht, in der (w) die Wachstumsrate der Nominallöhne, (prod) die der Produktivität, (p) die nationale Preissteigerung und (pEZB) das Preissteigerungsziel der Geldpolitik sind, soll gelten:

$$
\mathrm{w}-\operatorname{prod}=\mathrm{p}=\mathrm{pEZB}
$$

Diese Formel gibt die schon angesprochene Goldene Regel für die Lohn- und Preisentwicklung für jeden Mitgliedstaat in einer Währungsunion wieder. Die Einhaltung der „linken“ Gleichung führt dazu, dass die (primäre) Einkommensverteilung unverändert bleibt; die „rechte“ Gleichung gewährleistet, dass die nationale Preisentwicklung im Einklang mit dem Preisziel der EZB ist.

Diese Goldene Regel gilt für den Fall einer ausgeglichenen Ausgangsituation. Angesichts des noch notwendigen weiteren Abbaus noch bestehender überschießender

\section{ABBILDUNG 1}

\section{Veränderung der nominalen Lohnstückkosten (in Prozent) und der Leistungsbilanz (in Prozentpunkten des BIP)}

Angaben in Prozent und in Prozentpunkten des BIP
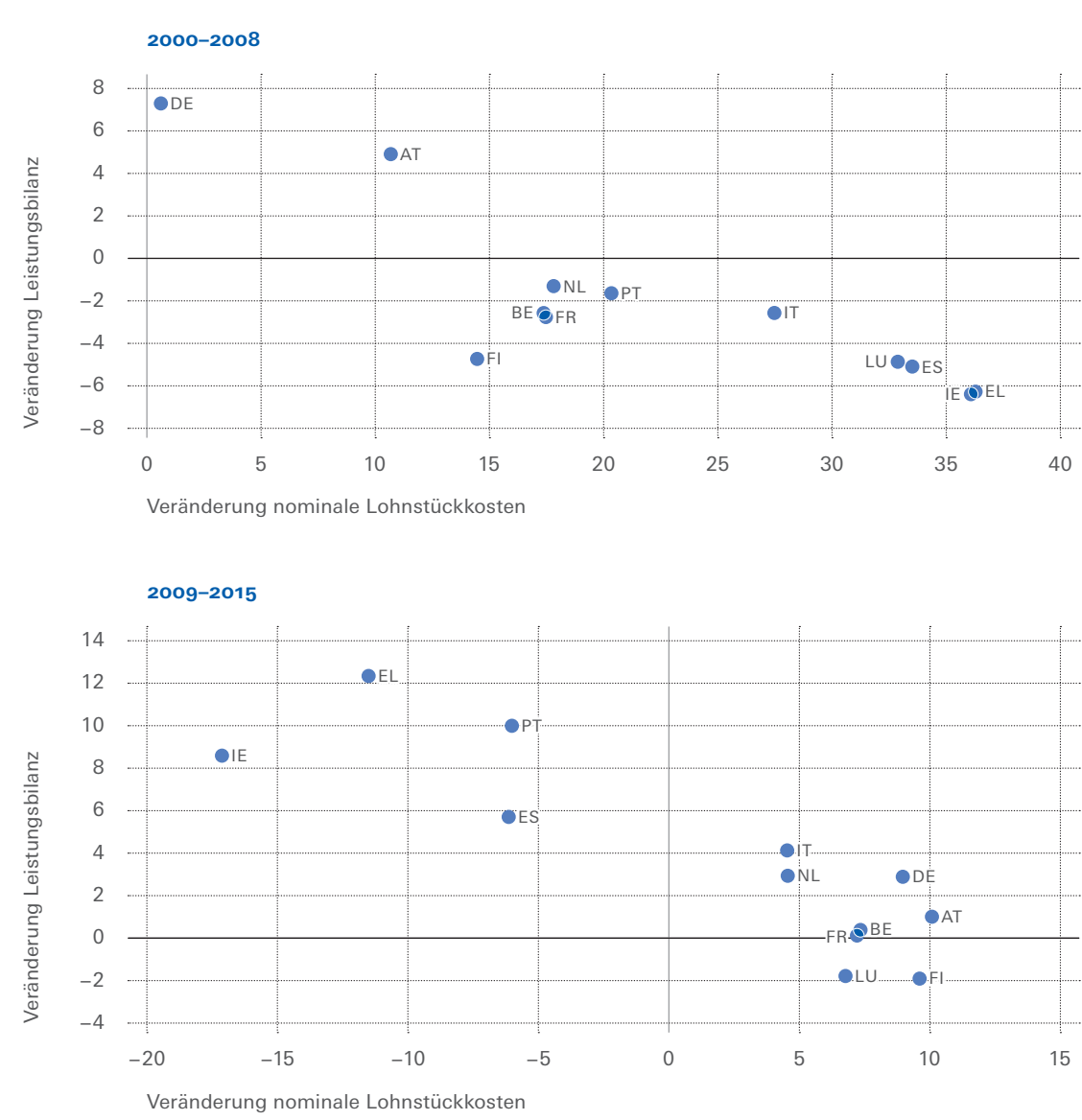

$\mathrm{AT}=$ Österreich, $\mathrm{BE}=$ Belgien, $\mathrm{DE}=$ Deutschland, $\mathrm{EL}=$ Griechenland, $\mathrm{ES}=$ Spanien, $\mathrm{Fl}=$ Finnland, $\mathrm{FR}=$ Frankreich $\mathrm{IE}=$ Irland; IT = Italien, $\mathrm{LU}=$ Luxemburg, $\mathrm{NL}=$ Niederlande, $\mathrm{PT}=$ Portugal .

Quelle: AMECO, eigene Berechnungen

4 Das heißt nicht, dass die Mitgliedstaaten nicht weiter versuchen sollen, ihre Produktivität zu steigern. Im Gegenteil: Gerade Mitgliedstaaten mit bisher relativ niedriger Produktivität sollen alles unternehmen, um ihre Produktivität so stark und schnell wie möglich zu erhöhen. Entscheidend ist, dass dabei die Nominallohnentwicklung im Einklang mit der Produktivitätssteigerung bleibt. 
gesamtwirtschaftlicher Divergenzen muss die Goldene Lohnregel bis zu einem befriedigenden Ausgleich modifiziert werden. Länder mit noch gravierenden Überschüssen müssen ihre Lohn-, Preis- und binnenwirtschaftliche Entwicklung beschleunigen, Länder mit Defiziten zur Goldenen Regel zurückkehren, nicht aber einen deflatorischen Kurs fortsetzen, der ihnen im Rahmen der sogenannten Programme aufgenötigt wurde.

\section{Stabilität der Einkommensverteilung}

Eine Verletzung der Goldenen Regel kann dadurch geschehen, dass in einem Mitgliedstaat die Preisentwicklung vom Preisziel der EZB abweicht. Sie kann aber auch dadurch erfolgen, dass die Preise nicht im Maße der Lohnstückkosten steigen, sondern sich stärker oder schwächer entwickeln. Es kommt zu einer Veränderung der realen Lohnstückkosten und damit in der primären Einkommensverteilung. Eine solche Veränderung wird überwiegend als ein Problem der Verteilungsgerechtigkeit angesehen. Sie ist aber auch ein Problem bei der Entstehung von zwischenstaatlichen Divergenzen. Verändert sich die Verteilung, verändern sich auch dadurch beeinflusste gesamtwirtschaftliche Aggregate, insbesondere Konsum und Sparen. Das wiederum schlägt durch auf Binnennachfrage, Import und Kapitalexport. Steigt z. B. die volkswirtschaftliche Gewinnquote, könnte das die Sparquote erhöhen und spiegelbildlich die Konsumquote senken. Dadurch könnten die Nettoexporte steigen, was wiederum auch durch einen Kapitalexport aus der erhöhten Ersparnis finanziert werden kann. Im Ergebnis würde dieser Prozess ebenfalls zur Ausbildung von Divergenzen bzw. Verstärkung bereits bestehender beitragen. Um eine dauerhafte Verschlechterung der Verteilung zulasten der Arbeitnehmer z. B. durch eine Vermachtung von Märkten zu vermeiden, müssen dem eine aktive Wettbewerbspolitik und eine Stärkung der Gewerkschaften entgegenwirken.

\section{Geld- und Fiskalpolitik für Wachstum und Beschäftigung}

Eine Lohnpolitik, die wie oben gefordert für Stabilität und Konvergenz sorgt, kann nicht zugleich auch Wachstum und Beschäftigung befördern. ${ }^{5}$ Das ist in erster Linie Aufgabe der Geldpolitik auf der Euroebene und der Fiskalpolitik auf der nationalen Ebene. Insbesondere wenn die konjunkturelle Entwicklung zu starke Ausschläge nach oben wie nach unten zeigt, muss die nationale Fiskalpoli- tik mit ihren Mitteln gegensteuern. Dazu stehen ihr sowohl die automatischen Stabilisatoren wie auch diskretionäre Maßnahmen zur Verfügung. Sollten die nationalen fiskalpolitischen Maßnahmen nicht ausreichen, müssen subsidiär solche auf Ebene der Eurozone hinzukommen.

Wenn die nationale Politik in einem geeigneten Mix aus Lohn- und Fiskalpolitik das Preisziel der Geldpolitik einhält, kann und muss - so der Vertrag - die Geldpolitik möglichst günstige monetäre Bedingungen für Investitionen und Beschäftigung bereitstellen. Dafür verfügt die Geldpolitik über vielfältige konventionelle Instrumente und in Ausnahmefällen, wie nach der Krise, auch über unkonventionelle Maßnahmen wie z.B. quantitative easing, also die unkonventionelle expansive Geldpolitik der EZB.

\section{Kooperation und Koordinierung im Dialog}

Wie die Vergangenheit zeigt, stellt sich eine derartig konsistente stabilitäts- und wachstumsorientierte Ausrichtung der gesamtwirtschaftlichen Politik nicht von selber ein. Gerade im Gefolge massiver außen- wie binnenwirtschaftlicher Schocks, wie z. B. die beiden Ölpreisschocks in den 1970er und 8oer Jahren, aber auch im Zuge der deutschen Wiedervereinigung, ist es im Gegenteil zu massiven Konflikten zwischen den gesamtwirtschaftlichen Instrumenten und ihren Akteuren mit der Folge eines rapiden Einbruchs der Konjunktur und eines massiven Anstiegs der Arbeitslosigkeit gekommen. Aber auch in guten Zeiten wäre bei einem kooperativen Verhalten ein besseres Ergebnis möglich gewesen.

Wegen ihrer fundamentalen Bedeutung für Wachstum, Beschäftigung und Stabilität ist daher Kooperation in Form der Koordination ein zentrales Element im Regelwerk von EU und WWU. „Die Mitgliedstaaten betrachten ihre Wirtschaftspolitik als eine Angelegenheit von gemeinsamem Interesse und koordinieren sie im Rat nach Maßgabe des Artikels 120“ (Art.121, 1 AEUv). Art.120 AEUv wiederum verweist zum einen auf die „Grundzüge der Wirtschaftspolitik der Mitgliedstaaten und der Union“ in Art. 121 als zentrales Instrument der Koordinierung, zum

5 Das wäre nur möglich, wenn die Löhne und mit ihr die Preise stark abgesenkt würden, sodass auch ohne Ausweitung der nominalen Geldmenge die reale Geldmenge entsprechend steigt - das bekannte "monetary management by the trade unions, aimed at full employment" (Keynes 1973, S. 267), das aber Keynes gerade wegen deflatorischer Risiken im Vergleich mit einer produktivitätsgerechten Nominallohnsteigerung, wie sie die Goldene Regel vorsieht und einer Ausweitung der nominalen Geldmenge nicht empfiehlt. 
anderen auf Art.119, in dem als Ziele der Koordinierung „stabile Preise, gesunde öffentliche Finanzen und monetäre Rahmenbedingungen sowie eine dauerhaft finanzierbare Zahlungsbilanz" genannt werden. Art.120 AEUV stellt zugleich die Verbindung zu Art. 3 Euv her, der eine "nachhaltige Entwicklung Europas auf der Grundlage eines ausgewogenes Wirtschaftswachstums und von Preisstabilität, eine im hohen Maße wettbewerbsfähige soziale Marktwirtschaft, die auf Vollbeschäftigung und sozialen Fortschritt abzielt, sowie ein hohes Maß an Umweltschutz und Verbesserung der Umweltqualität" einfordert. Die einzelnen Schritte der Koordination werden in den weiteren Absätzen des Art. 121 beschrieben.

Inzwischen ist das Verfahren der wirtschaftspolitischen Koordination deutlich vertieft und erweitert worden. Neben der Reform des Stabilitäts- und Wachstumspaktes (SWP) ist hier insbesondere die Verordnung zur Vermeidung und Korrektur makro-ökonomischer Ungleichgewichte zu nennen. Mit dem Europäischen Semester wurde ein Rahmen geschaffen, in dem vielfältige Koordinierungsinstrumente nach Inhalt und Ablauf aufeinander abgestimmt werden. Neben der Haushaltsüberwachung sind das im Zusammenspiel von Mitgliedstaaten und Euroraum auf analytischer Ebene der Jährliche Wachstumsbericht und die Länderberichte, auf politischer Ebene die Empfehlungen für den Euroraum sowie die Nationalen Reformprogramme und die Länderspezifischen Empfehlungen, die sich ihrerseits an den Integrierten Leitlinien, d.h. den Grundzügen und den Beschäftigungspolitischen Leitlinien orientieren.

Art.121 Aeuv sieht in Falle eines Verstoßes gegen die Regeln der Koordinierung Sanktionen vor. Sie beinhalten derzeit eine „Verwarnung“, begleitet von „Empfehlungen“, die auf Ratsbeschluss auch veröffentlicht werden können. Derartige Sanktionen können aber nicht die Wirkung haben, die sie zur Durchsetzung eines so zentralen Elements wie der Koordinierung haben müssten. Deutlich effektiver dürften hier z. B., wie bereits von anderer Seite vorgeschlagen, die Sperrung des Zugangs zu EU-Fonds oder anderen fiskalischen Instrumenten sein (European Parliament 2016). Auch eine Beschränkung von Mitwirkungsrechten könnte in Betracht gezogen werden, um die wirtschaftspolitische Koordinierung durch die Mitgliedstaaten so durchzusetzen (compliance), wie es ihrer Bedeutung zukommt.

In der konkreten Umsetzung der Koordinierung sollen sich die Vertreter von Geld-, Fiskal- und Lohnpolitik über die aktuelle Wirtschaftslage und deren Perspektiven austauschen. Auf dieser gemeinsamen Erkenntnisgrundlage sollen sie die sich daraus ergebenden wirtschaftspolitischen Herausforderungen identifizieren. Schließlich soll jeder Akteur seinen Beitrag zu deren Bewältigung und seine Erwartung über die Beiträge der jeweils anderen Akteure benennen.

Dieser Austausch hat die Autonomie und Unabhängigkeit der einzelnen Politikbereiche zu respektieren.
Deshalb soll er auch in Form eines Dialogs stattfinden. Dieser ermöglicht unmittelbares Signalisieren und sofortige Rückkopplungen und wirkt vertrauensbildend. Entscheidungen trifft dann jeder Akteur in seinem Bereich zwar unabhängig, aber im Lichte des vorangegangen Austauschs und unter Abwägung bzw. Berücksichtigung der Rückwirkungen nationaler Entscheidungen auf die anderen EU-Länder. Und nur wenn jeder seinen Beitrag zu einem konsistenten, widerspruchsfreien Policy-Mix leistet, erntet auch jeder die Früchte, die nur aus dieser Kooperation erwachsen. Aus dieser Einsicht sollte dann auch ein starkes Motiv zur Einhaltung der von allen Akteuren für richtig erachteten Ausrichtung der jeweiligen Politik und zu ihrer Fortsetzung erwachsen.

Eine wichtige Voraussetzung für das Gelingen einer solchen Kooperation der wirtschaftspolitischen Akteure ist, dass ihr Handeln von einer tragfähigen Analyse und Prognose der gesamtwirtschaftlichen Entwicklung und politischen Erfordernisse geleitet wird. Es empfiehlt sich daher, dass die politischen Entscheidungsprozesse durch wissenschaftliche Politikberatung unterstützt werden. Dies könnte ein Gremium von Sachverständigen leisten, das im Vorfeld politischer Entscheidungsprozesse mögliche Entwicklungs- und Handlungsszenarien erarbeitet. Dabei muss ökonomische Komplexität in quantitativ nachvollziehbare sowie politisch verständliche und umsetzbare Handlungsoptionen übersetzt werden. Diese werden dann von den politischen Akteuren bewertet und - falls für zielführend erachtet - umgesetzt. Wegen ihres Gewichts für die politische Entscheidungsfindung muss diese Expertise auf der Basis neutraler, pluraler und unabhängiger Berater erfolgen, um eine Verengung der Expertise auf eine einzige Theorierichtung zu vermeiden.

\section{Nachhaltige Wirksamkeit durch Institutionalisierung}

Um nachhaltig wirksam zu sein, müssen die oben entwickelten Verfahren in stabile, praxisnahe Formen institutionalisiert werden. Dies gilt für die nationale wie die Ebene des Euroraums. Dabei sollen aus Praktikabilitätsgründen Vertragsänderungen vermieden werden. Ebenso soll angesichts der großen Zahl bereits bestehender Institutionen und Prozesse auf neue verzichtet werden. Die vorhandenen Institutionen könnten genutzt und ihre Agenda so angepasst werden, dass sie die Umsetzung der zuvor beschriebenen Anforderungen leisten können.

Schließlich soll eine zielführende Subsidiarität eingehalten werden: Was auf nationaler Ebene zur Erreichung der Ziele geleistet werden kann, soll vorab und dort erfolgen. Falls das nicht ausreicht, sollen auf Ebene der Eurozone vorhandene Instrumente genutzt bzw. zusätzliche 
Instrumente bereitgestellt werden. Für die notwendige Institutionalisierung wird nachstehend ein konkreter Vorschlag unterbreitet (eine detailliertere Darstellung findet sich in Koll/Watt 2018a, b).

\subsection{Nationale Ebene}

(1) Erweiterung der Aufgaben der Nationalen Ausschüsse für Produktivität um die Analyse und Prognose der gesamtwirtschaftlichen Entwicklung und um die Erarbeitung von Politikszenarien, möglichst in quantitativer Form, zur Bewältigung der erkennbaren Herausforderungen auf nationaler Ebene. Das bisherige Mandat sieht bereits wichtige makroökonomische Ansatzpunkte für eine derartige Ausweitung vor.

(2) Einrichtung eines Gremiums zur Verbesserung der Kooperation zwischen Fiskal- und Lohnpolitik sowie der makroprudenziellen Politik der nationalen Zentralbank auf der Basis der Expertise des oben skizzierten Beratergremiums. Dabei sind die geldpolitischen Vorgaben durch die EZB zu berücksichtigen. Die Struktur dieses Gremiums könnte der des bestehenden Makroökonomischen Dialogs auf EU-Ebene nachgebildet werden, d.h. unter Teilnahme von Vertretern der Sozialpartner, der nationalen Fiskalpolitik und der nationalen Vertreter des Europäischen Systems der Zentralbanken (ESZB). Zu prüfen ist, inwieweit die Nationalen Fiskalräte (Fiscal Councils) diese Aufgabe übernehmen können. Die Ergebnisse dieses Gremiums sollen insbesondere bei der Formulierung der Nationalen Reformprogramme und der Umsetzung der Länderspezifischen Empfehlungen berücksichtigt werden.

\subsection{Euroraum}

(1) Erweiterung der Aufgaben und des Personals des Beratenden Europäischen Fiskalausschusses um die Analyse und Prognose der gesamtwirtschaftlichen Entwicklung und um die Erarbeitung von (quantifizierten) Politikszenarien zur Bewältigung der erkennbaren Herausforderung auf Ebene der Eurozone

(2) Einbezug der Vertreter der europäischen Spitzenverbände der Sozialpartner in die Beratungen der Eurogruppe, in der die Finanzminister aller Mitgliedstaaten und die EZB anwesend sind. Das könnte informell geschehen und versuchsweise zweimal jährlich vor oder während der regelmäßigen Sitzungen erfolgen. Dieses informelle und erweiterte Format sollte zeitlich so angesiedelt werden, dass das Gremium im Rahmen des Europäischen Semesters einen Beitrag zu den Beratungen über die Empfehlungen für die Eurozone, zur Prüfung der Nationalen Reformprogramme und zur Formulierung der Integrierten Leitlinien und der Länderspezifischen Empfehlungen einbringen kann.
(3) Denkbar wäre auch eine Umgestaltung des existierenden Makroökonomischen Dialogs auf EU-Ebene. Hierbei kommt es insbesondere darauf an, dass wegen der Bedeutung der nationalen Ebene anders als bisher die Finanzminister aller Mitgliedstaaten, zumindest die des Euroraums, dort präsent sind. Ferner sollte der Vorsitz nicht alle halbe Jahre mit der Ratspräsidentschaft wechseln, sondern ein mehrjähriger Vorsitz aus den Reihen des Rates oder der Kommission bestimmt werden. Ein solcher Makroökonomischer Dialog speziell für die Eurozone ist nicht zuletzt auch von den Präsidenten der EU gefordert worden (Juncker et al. 2015).

\section{Stärkung der Sozialpartner}

Die Lohnsetzungsstrukturen in den einzelnen Mitgliedstaaten der WWU sind sehr unterschiedlich. ${ }^{6}$ Es ist nicht notwendig, sie zu vereinheitlichen. Entscheidend ist, dass jedes Land die Goldene Preis- und Lohnregel einhält und seinen wirtschaftspolitischen Kurs gegebenenfalls entsprechend nachjustiert. Grundsätzlich kann hier eine Kombination von lohnpolitischen und fiskalischen Maßnahmen zum Tragen kommen.

In Mitgliedstaaten mit starken Systemen kollektiver Lohnfindung können und müssen die Sozialpartner eine gesamtwirtschaftlich relevante Rolle bei der Vermeidung von Ungleichgewichten sowie bei der Stabilisierung und Verstetigung der konjunkturellen Dynamik spielen, indem sie die Auswirkungen ihrer Abschlüsse auf die Gesamtwirtschaft berücksichtigen. Dort, wo es kaum gesamtwirtschaftlich relevante sozialpartnerschaftliche Strukturen gibt, die Lohnfindung überwiegend dezentral stattfindet und diese wesentlich von der konjunkturellen Dynamik abhängt, muss die Stabilisierungsfunktion weitgehend von der Fiskalpolitik übernommen werden.

Der Einsatz der Fiskalpolitik kann dabei aber in Konflikt geraten mit bestehenden fiskalischen Regeln, wie z. B. mit der Schuldenbremse in Deutschland oder mit dem Stabilitäts- und Wachstumspakt der EU - Regeln, die wir für reformbedürftig halten. Fiskalpolitische Erfordernisse können auch den subsidiären Einsatz von Mitteln auf Ebene des Euroraums erfordern. Eine solche Hilfe kann die beschlossene Stärkung des Europäischen Stabilitätsmechanismus (ESM) mit sich bringen. ${ }^{7}$ Hierdurch sollen

6 Übersichten gibt es u.a. bei Visser (2013); Watt (2017, S. 82ff.); siehe auch Koll (2013, S.63ff.).

7 Vgl. hierzu den jüngsten Bericht der Eurogruppe vom 3. Dezember 2018: www.consilium.europa.eu/de/meetings/ eurogroup/2018/12/03/ und dessen Billigung durch den Europäischen Rat vom 14. Dezember 2018: www.consilium. europa.eu/de/meetings/euro-summit/2018/12/14/ 
Länder unterstützt werden können, die zwar eine gute gesamtwirtschaftliche Entwicklung, aber keinen ausreichenden Zugang zu den Finanzmärkten mehr haben. Wie schon bisher ist jedoch die Vergabe der Mittel an strikte Reform- und Sparauflagen geknüpft. Hilfe könnte auch ein Eurozonen-Budget oder eine europäische Rückversicherung der nationalen Systeme zur Sicherung bei Arbeitslosigkeit leisten; allerdings sind hier Umfang und Finanzierung nach wie vor umstritten.

Im Ergebnis sollte eine Stabilisierung durch diskretionäre Fiskalpolitik möglichst weitgehend durch die Einhaltung der Goldenen Lohn- und Preisregel, aber auch durch den Ausbau nationaler automatischer Stabilisatoren (vgl. Watt 2011) überflüssig gemacht werden.

Daher sollten alle Länder und auch die europäischen Institutionen ein gemeinsames Interesse an dem Ausbau sozialpartnerschaftlicher Strukturen in den Mitgliedstaaten haben. In dieser Absicht bestärken sollte sie ein zunehmender Konsens in der wissenschaftlichen Diskussion darüber, dass - auch unabhängig von den spezifischen Anforderungen der WWU koordinierte Lohnverhandlungsstrukturen vorteilhaft für die gesamtwirtschaftliche Entwicklung sind (OECD 2018; European Commission 2017a; Watt 2017; Koll 2013, S. 62ff.).

Im spezifischen Fall der WWU würde eine stärkere Koordinierung der Lohnfindung zwischen den Mitgliedstaaten Gewicht und Rolle der Sozialpartner in der economic governance auf der Ebene der Eurozone stärken. Eine derart abgestimmte Lohnpolitik kann dann auf Augenhöhe mit der Geld- und Fiskalpolitik agieren und zu einem stabilitäts-, wachstums- und beschäftigungsorientierten makroökonomischen Policy-Mix beitragen und ihrerseits den notwendigen Beitrag der anderen Akteure einfordern.

Als Teil des Reformprozesses mit dem Ziel einer Vertiefung und Konvergenz der WWU sollte daher in geeigneter Form in die Errichtung und Stärkung makroökonomisch wirksamer sozialpartnerschaftlicher Strukturen als capacity building investiert werden. Eine derartige Stärkung der Sozialpartner wird nunmehr auch von der Europäischen Kommission im Rahmen ihrer Empfehlung zum Europäischen Pfeiler der Sozialen Rechte und einer ergänzenden Mitteilung unterstützt (European Commission 2017 b c). Die Sozialpartner, und nicht zuletzt die Gewerkschaften auf nationaler wie europäischer Ebene, sollten hierin eine Chance sehen, sich als wichtige wirtschaftspolitische Akteure zu profilieren.

\section{Fazit}

Schon vor der Krise im Euroraum hat es eine Vielfalt an Vorschlägen, Reformen, neuen Regelungen und Institutionen auf der Ebene der EU und der WWU gegeben. Sie alle haben nicht ausgereicht, um die Krise zu verhindern, oder waren sogar kontraproduktiv. Verhindert werden muss aber in jedem Fall eine Wiederholung eines derart schockartigen Einbruchs der wirtschaftlichen Entwicklung in vielen Mitgliedstaaten. Mit diesem Ziel sind zwar seit der Krise erneut vielfältige Vorschläge zur Vertiefung und Stabilisierung insbesondere des Euroraums unterbreitet worden; allerdings orientieren sie sich mehrheitlich auf die Stabilisierung des Bankenund Finanzmarktsektors. Andere zielen auf eine fiskalische Stabilisierung, wenn Mitgliedstaaten bei dieser Aufgabe national überfordert sind.

Aber so nützlich solche Intentionen im Einzelnen sein mögen, so wenig sind sie allein geeignet, eine erneute Krise zu vermeiden oder gar eine nachhaltige Prosperität der Eurozone zu gewährleisten. Wenn die Krise auch nur einen einzigen Sinn gehabt haben soll, dann den, die richtigen Fragen nach ihrer Ursache zu stellen und aus der Analyse die richtigen Antworten zu finden. Unsere Antwort liegt

- in einer Stärkung von Kompetenz und Verantwortung (ownership) durch Einhaltung der Goldenen Regel der Preis- und Lohnentwicklung auf nationaler Ebene;

- im subsidiären Einsatz alter und neuer Instrumente hierzu auf Ebene der Eurozone;

- mit dem Ziel eines stabilitäts-, wachstums- und beschäftigungsorientierten makroökonomischen Policy-Mix,

- ergänzt und unterstützt durch zielführende strukturelle Reformen;

- unter entsprechender Erweiterung der Agenda und Mitgliedschaft bestehender Institutionen auf nationaler und Eurozonenebene;

- zur Bewältigung dieser wirtschaftspolitischen Herausforderungen als optimale Verbindung von Risikovermeidung und Risikoteilung.

Es reicht nicht aus, das europäische Dach über den Wohnungen nationaler Mitgliedstaaten neu zu decken, wenn nicht gleichzeitig auch deren makroökonomische Fundamente verstärkt werden.

\section{LITERATUR}

Bénassy-Quéré, A. / Brunnermeier, M. / Enderlein, H./ Farhi, E./ Fratzscher, M. / Fuest, C. / Gourinchas, P./ Martin, Ph./ Pisani-Ferry, J. / Rey, H. / Schnabel, I. / Véron, N. / Weder di Mauro, B. (2018): Reconciling risk sharing with market discipline: A constructive approach to euro area reform: Center for Economic Policy Research, Policy Insights No. 91, January 2018

Bibow, J./ Flassbeck, H. (2018): Das Euro-Desaster, Frankfurt a. M.

Dullien, S. (2018): Wie weiter mit der Euro-Zone? Aktuelle Reformvorschläge im Vergleich: Friedrich Ebert Stiftung, WISO Diskurs 201/2018, Bonn

European Commission (2010): Directorate-General for Economic and Financial Affairs, Co-ordination of economic policies in the EU, Quarterly Report on the

Euro Area 9 (1), Brussels

European Commission (2017a): European Semester Thematic Factsheet, Wage Developments and Wage Setting Systems, Brussels

European Commission (2017b): Commission recommendation on the European pillar of social rights, Brussels, 26.4.2017

European Commission (2017c): Communication from the Commission to the European Parliament, the Council, the European Economic and Social Committee and the Committee of the Regions, Brussels, 26.4. 2017

European Parliament (2016): Draft Report, On possible evolutions of and adjustments to the current institutional set-up of the European Union (2014/2248(INI), Committee on Constitutional Affairs, PR\1100144 En.doc, 5. 7.2016

Herr, H. / Priewe, J./ Watt, A. (2019): Still time to save the Euro. A new agenda for growth and jobs with a focus on the Euro area's four largest countries, Social Europe Publishing, Berlin

Juncker, J.-C./ Tusk, D. / Dijsselbloem, J. / Draghi, M. / Schulz, M. (2015): Die Wirtschafts- und Währungsunion vollenden, Bericht der fünf Präsidenten vom 22. 6. 2015, www.ecb.europa.eu/pub/pdf/other/5presidentsreport.de.pdf

Keynes, J.M. (1973): The general theory of employment, interest and money, edition 1973, Cambridge

Koll, W. (2013): Neue Wirtschaftsregierung und Tarifautonomie in der Europäischen Union, Makroökonomische Koordinierung im Dialog: Institut für Makroökonomie und Konjunkturforschung (IMK), Study 30, März

Koll, W. (2016): Vom Stabilitäts- und Wachstumsgesetz zum Wohlstands- und Nachhaltigkeitsgesetz, in: Wirtschaftsdienst 96 (1), S. 40-48

Koll, W./ Watt, A. (2018): Vertiefung und Konvergenz der Europäischen Wirtschafts- und Währungsunion durch konzeptionelle und institutionelle Reformen der makroökonomischen Koordinierung: Institut für Makroökonomie und Konjunkturforschung (IMK), Study 61, Juni 
Koll, W./ Watt, A. (2018): Convergence and stability in the Euro area through effective macroeconomic policy coordination:Macroeconomic Policy Institute (IMK), Study 61e, August

OECD (Organisation for Economic Co-operation and Development) (2018): Employment Outlook, Paris

Regling, K. (2010): Interview „Rettungsfonds - Der Bund verdient eine ordentliche Marge", in: Berliner Zeitung vom 23./24.11. 2010

Visser, J. (2013): Wage bargaining institutions. From crisis to crisis: European Commission, Economic Papers 488, April

Watt, A. (2011): Strengthening the automatic stabilisers in Europe: Why, what and how?, in: Niechoj, T. / Onaran, Ö. / Stockhammer, E. / Truger, A. / van Treeck, T. (Hrsg.): Stabilising an unequal economy? Public debt, financial regulation and income distribution, Marburg, S. 197-220

Watt, A. (2017): Explaining unemployment developments in Europe: the role of wage-setting institutions and macroeconomic policies: Macroeconomic Policy Institute (IMK), IMK Study 57, Düsseldorf

Watt, A. / Watzka, S. (2018): Overcoming Euro area fragility: Macroeconomic Policy Institute (IMK), Report 139, Düsseldorf

\section{AUTOREN}

WILLI KOLL, Dr., war Ministerialdirigent im Bundesministerium für Wirtschaft und Energie und im Bundesministerium der Finanzen. Er war langjähriges Mitglied im Wirtschaftspolitischen Ausschuss der EU und der OECD sowie im Makroökonomischen Dialog der EU

@ hwkoll@t-online.de

ANDREW WATT, Dr., ist Referatsleiter für Europäische Wirtschaftspolitik im Institut für Makroökonomie und Konjunkturforschung (IMK) der Hans-BöcklerStiftung.

andrew-watt@boeckler.de 